\title{
The influence of regional anaesthesia and local anaesthetics on cardiac repolarization
}

\author{
Jowita Biernawska ${ }^{1}$, Jarosław Kaźmierczak ${ }^{2}$, Katarzyna Kotfis ${ }^{1}$, Maciej Żukowski ${ }^{1}$ \\ ${ }^{1}$ Department of Anaesthesia, Intensive Care and Acute Poisoning, Pomeranian Medical University, Szczecin, Poland \\ ${ }^{2}$ Department of Cardiology, Pomeranian Medical University, Szczecin, Poland
}

\begin{abstract}
The normal function of the heart muscle is the result of electro-mechanic and hemodynamic coupling. Modification of the structure and activity of ion channels within the cardiomyocytes may induce cardiac arrhythmias. Electrophysiological mechanisms of arrhythmia, generated by a prolonged period of repolarization, result either from conduction disturbances (reentry mechanism) and/or the induction of beats (early after-depolarizations). Local anaesthetic drug deposition does not affect the repolarization period, as long as the concentration of the free drug in the plasma does not reach the critical value to cause toxic effects in the cardiomyocytes.

When analyzing the effect of regional anaesthesia on the repolarization period it is essential to acknowledge the activity or blockade of adrenergic fibres. Blocking the sympathetic fibres', including level T1 to T4, leads to a shortening of the QT interval and a reduction of QT dispersion. Adrenergic blockade as a result of spinal anaesthesia causes severe adrenergic activity above the level of the block and therefore prolongs repolarization. Stellate ganglion block on the right side causes a significant prolongation of the QT interval and QT dispersion.

Regardless of the reasons for prolongation of the repolarization period (congenital or acquired), vigilance is required within the perioperative anaesthetic management of a patient, so as not to lead to the occurrence of ventricular arrhythmias. Regional anaesthesia techniques and properly used local anaesthetic drugs are regarded as being safe in these patients.
\end{abstract}

Key words: long QT syndromes, cardiac repolarization, regional anaesthesia, local anaesthetics

\section{CARDIAC ELECTROPHYSIOLOGY}

Normal function of the heart muscle is the result of electro-mechanic and haemodynamic coupling. When considering cardiac electrical activity from the point of view of a working myocardial cell, the following must be acknowledged: the relaxed state (polarization of the cell membrane) and active state (depolarization-repolarization) occurring after activation by the conduction system. Changes in the phases of action potential and the resting potential are the result of a synchronized movement of ionic currents through the ion channels, pumps and cardiomyocyte cell membrane carriers. The period of depolarization is initiated by an inward sodium current $\left(I_{\mathrm{Na}}\right)$, supported by an inward calcium current $\left(\mathrm{I}_{\mathrm{Ca}-\mathrm{L}}\right)$. During repolarization the flow of current occurs through the channels conducting the potassium current $\left(\mathrm{I}_{\mathrm{Kr}^{\prime}} \mathrm{I}_{\mathrm{Ks}^{\prime}} \mathrm{I}_{\mathrm{to}} \mathrm{I}_{\mathrm{K} \text { (ATP) }}\right)$ and to a lesser extent by the sodium current $\left(\mathrm{I}_{\mathrm{Na}}\right)$, calcium current $\left(\mathrm{I}_{\mathrm{Ca}-\mathrm{L}}\right)$ and non-selective cation channel $\left(\mathrm{I}_{\mathrm{NS}}\right)$. This is depicted in Figure 1.

The intracellular influx of calcium ions during the plateau phase enables a calcium dependent calcium release from the sarcoplasmic reticulum, leading to a contraction of the working myocardial cell. Under such physiological conditions during repolarization, potassium $\mathrm{I}_{\mathrm{Kr}}$ and $\mathrm{I}_{\mathrm{Ks}}$ currents play a key role in deciding the proper course of this phase of the action potential. In contrast, prolonged sodium and calcium currents slow down the cardiac repolarization process, therefore creating an environment for the occurrence of ventricular arrhythmias [1]. The concept of "repolarization reserve" has been described by Roden [2]. The loss of one component (one of the potassium currents) ordinarily 


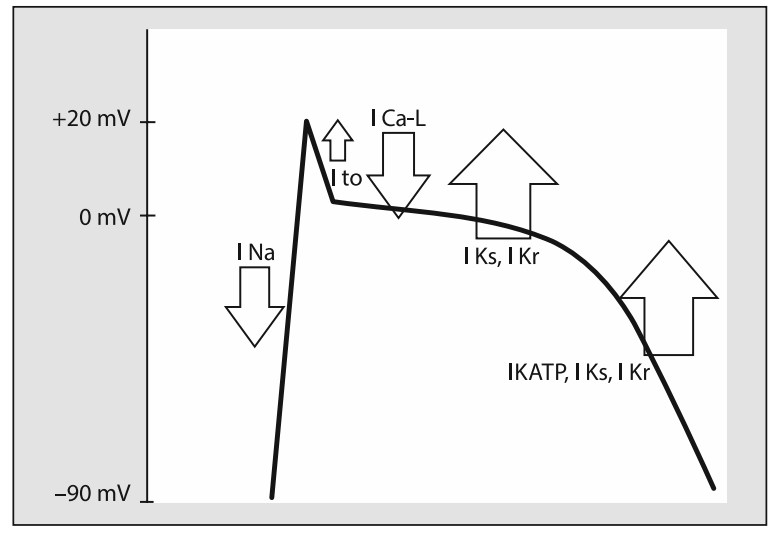

Figure 1. Ion currents and action potential in cardiomyocyte (arrow $\downarrow$ inward current, arrow $\uparrow$ outward current)

will not lead to failure of repolarization (i.e., marked QT prolongation), because of amplification of the remaining ion currents.

\section{ELECTROCARDIOGRAPHIC ASSESSMENT OF THE REPOLARIZATION PERIOD}

The normal sequence of the action potential phases can be determined indirectly on a electrocardiogram (ECG). The clinically most useful indicators of the electrophysiological assessment of the repolarization phase are as follows: QT interval, the length of the QT interval corrected to the frequency of the rhythm (QTc), QT interval dispersion, T wave morphology changes and the transmural dispersion of repolarization (TDR), which is expressed in the precordial ECG leads as the interval measured from the peak of the T wave to its end (Tpeak-Tend, Tp-e) [3, 4]. The QT interval typically is measured by lead II of a 12 lead ECG from the onset of the QRS complex to the point at which the T wave ends. There is an American Heart Association recommendation regarding how to correct the QT interval in the case of a QT interval prolongation in ventricular conduction defects. This can be best accomplished by incorporating QRS duration and RR interval as covariates into the QT-adjustment formula or by using the JT interval (QT duration-QRS duration). If the JT interval is chosen, normal standards established specifically for the JT interval should be used [5]. Under physiological conditions, the QT interval depends mainly on the age, gender and heart rate. The normal range for the rate-corrected QT interval is similar in females and males from birth until late adolescence. In adults, men have slightly shorter QT intervals than females. Corrected QT intervals of more than $0.46 \mathrm{~s}$ in females, and more than $0.45 \mathrm{~s}$ in men are considered prolonged. This difference decreases with age, as the plasma level of testosterone is correlated with age, so the influence testosterone on the calcium current is decreased [5]. As the QT interval also depends on the heart rate, a correction for the heart rate must be made. A useful method in order to correct the current value of the QT interval is to use one of the available formulas (i.e., Bazett's, Fridericia's, Ashman's, Hodges' and the Framingham formula,). However, there is no general consensus on the best formula to be useful in clinical practice. . Physiological QT dispersion indicates the variable values of the QT interval length in different ECG leads (QT max - QT min). The correct value is less than $50 \mathrm{~ms}$. QT dispersion values greater than $100 \mathrm{~ms}$ can be considered as potentially arrhythmogenic [6, 7]. The transmural dispersion of repolarization (TDR) has a recognized prognostic value in relation to the arrhythmogenic potential of polymorphic ventricular tachycardia (namely torsade de pointes, $\mathrm{TdP}$ ) and sudden cardiac death (SCD). Under physiological conditions the fastest repolarization occurs in the cells of the epicardium, while the slowest repolarization takes place in the middle of the myocardial cells. The T peak corresponds to the total repolarization of the epicardial cells and "T end" point - determines the return of excitability of the entire myocardium. The normal value for Tp-e is $40-110 \mathrm{~ms}$ and its prolongation above $117 \mathrm{~ms}$ is a recognized marker of susceptibility to TdP [8-10]. Repolarization period disorders are defined as arrhythmogenic diseases caused by the disturbed function of ion channel subunits or the proteins that regulate them. The repolarization period can be longer, shorter or the beginning of repolarization can be earlier than normal. Primary electrical disturbances include congenital or acquired long QT syndrome, Brugada syndrome (pseudo RBBB and persistent ST segment elevation in leads V1-V3), catecholaminergic polymorphic ventricular tachycardia, congenital or acquired short QT syndrome (QTc $<0,3 \mathrm{~s})$ and early repolarization syndrome.

\section{THE MECHANISM OF ARRHYTHMIA FORMATION IN DISORDERS OF REPOLARIZATION}

Any modification of the structure and activity of the described channels and channel-related proteins (A kinase anchoring protein, AKAP) can induce arrhythmias. Electrophysiological mechanisms of arrhythmia result from conduction disturbances and/or the formation of beats. Conduction abnormalities occur in the type of reentry in which unidirectional conduction block coexists with the heterogeneous duration of refraction. They are usually initiated by an additional ventricular beat (premature ectopic depolarization) which occurs during repolarization (the incomplete return of sodium channel activity after previous excitation). The ECG picture shows that the additional stimulation occurs during the $\mathrm{T}$ wave (the so-called "R on $\mathrm{T}^{\prime \prime}$ phenomenon).

Mechanisms interfering with the formation of beats arise from pathological automaticity or triggered activity 
Table 1. Congenital long QT syndromes

\begin{tabular}{|c|c|c|}
\hline LQTS & Gene & Phenotype effect \\
\hline LQTS 1 & KCNQ1 (KVLQT1) & alfa subunit of the delayed rectifier potassium channel $\mathrm{I}_{\mathrm{Ks}}-$ slowly activating \\
\hline LQTS 2 & $\mathrm{KCNH} 2(\mathrm{HERG}) *$ & alfa subunit of the delayed potassium rectifier channel $\mathrm{I}_{\mathrm{Kr}}$ - rapidly activating \\
\hline LQTS 3 & SCN5A & Sodium channel $\mathrm{I}_{\mathrm{Na}}$ \\
\hline LQTS 4 & Ankyrin-B & Ankyrin B protein responsible for normal function of the $\mathrm{Na}^{+} / \mathrm{Ca}^{++}$exchanger, $\mathrm{Na}^{+} / \mathrm{K}^{+}$ATP-ase pump \\
\hline LQTS 5 & KCNE1 (minK) & beta subunit of the delayed potassium rectifier channel $\mathrm{I}_{\mathrm{Ks}}$ \\
\hline LQTS 6 & KCNE2 (MiRP1) & beta subunit of the delayed potassium rectifier channel $\mathrm{I}_{\mathrm{Kr}}$ \\
\hline LQTS 7 & KCNJ2 & inward rectifier potassium channel KIR2.1 \\
\hline LQTS 8 & CACNA1C & Calcium channel $\left(\mathrm{I}_{\mathrm{Ca}-\mathrm{L}}\right)$ \\
\hline LQTS 9 & CAV3 & caveolin 3, sodium channel $\mathrm{I}_{\mathrm{Na}}$ \\
\hline LQTS 10 & SCN4B & Sodium channel $\mathrm{I}_{\mathrm{Na}}$ beta 4 subunit \\
\hline LQTS 11 & AKAP9 & A-kinase anchoring protein 9 \\
\hline LQTS 12 & SNTA1 & alfa-1 syntrophin \\
\hline LQTS 13 & KCNJ5 & alfa subunit of the delayed potassium rectifier channel $\mathrm{I}_{\mathrm{Kr}}$ \\
\hline
\end{tabular}

* HERG (human ether-a-go-go-related gene) is a widely accepted equivalent of a cloned gene encoding the alpha subunit of the $\mathrm{I}_{\mathrm{Kr}}$ potassium channel

in the form of after-depolarizations. After-depolarizations are the result of the oscillation of the membrane potential, following the previous accumulation phase of the action potential (early - during repolarization, late - after repolarization). The ionic basis for early after-depolarizations (EADs), which begins at the plateau phase of the action potential, is the reactivation of the calcium current through L-type channels. On the other hand, within the EADs, which begin in phase 3 of the action potential, the inducing mechanism is a sodium-calcium exchanger current and the sodium current [11]. Variable expressions of sodium channels in different parts of the myocardium and the potassium current flow modification coexist with the inhomogeneous return of excitability (dispersion of repolarization), which is a substrate for the development of arrhythmias. The basic phenomenon that favours the induction of EAD is prolongation of the ventricular action potential. Electrocardiograms show a prolongation of the QT interval $[12,13]$. In contrast, the same EAD may underlie the occurrence of polymorphic ventricular tachycardia (TdP) and SCD, with the image being mainly due to a significant transmural dispersion of repolarization. There is also a dispersion of repolarization as a result of electrical remodelling, which results from hypertrophy or ventricular myocardial ischemia, or during adrenergic stimulation.

\section{LONG QT SYNDROME}

Prolongation of the QT interval may be congenital or acquired. Although the precise incidence of congenital QT prolongation is not known, it is estimated to be 1:2000-3000 live births. Modifications of the structure of ion channels leading to the prolongation of repolarization results from single nucleotide polymorphism (SNP) or the mutations of single genes. The genetic background is complex, and the list of candidate genes is still open [11]. Table 1 shows the congenital long QT syndromes identified thus far [14]. The most common genetic mutations that cause QT prolongation have been repeatedly discussed in the literature. The first type of congenital long QT syndrome results from KCNQ1 mutation, which is the gene encoding the proteins of the slow component of the delayed rectifier potassium current channel $\left(\mathrm{I}_{\mathrm{KS}}\right)$. The second type, resulting from $\mathrm{KCNH} 2$ mutation which disrupts the flow of the rapid component of the delayed rectifier potassium current channel $\left(\mathrm{I}_{\mathrm{Kr}}\right)$, occurs most frequently.

Hundreds of mutations have been identified in the 13 genes associated with the susceptibility to QT prolongation. While more than $75 \%$ of them are related to the LQTS $1-3$, about $1-2 \%$ correspond to LQTS 4 . Most commonly, the effect of the above-mentioned mutations is the lengthening of repolarization as a result of the inactivation of potassium channels (extended potassium current flow) or excessive activation of sodium channels ("late" sodium current). Normally, after a brief opening at the beginning of the action potential, the sodium channel is inactivated, carrying only a trace sodium current during the repolarization plateau. Mutations in genes encoding structural proteins of the sodium channel, as well as medicines, toxins, inflammatory agents, ischemia and oxidative stress can cause the destabilization of the period of inactivation. This leads to an increase in the flow of sodium ions (the so-called "late" sodium current), resulting in the disruption of sodium-calcium homeostasis $\left(\mathrm{Na}^{+} / \mathrm{Ca}^{++}\right.$exchanger), which is reflected by an increase in intracellular calcium during diastole. The flow of "late" sodium current is not uniform in all cells, so the dispersion of the action potential is observed, along with the increased, 
pathological excitability of the working cardiomyocytes, triggered activity (EADs) and the substrate for reentry. The presence of the pathological "late" sodium current also contributes to the prolongation of the action potential resulting from the blocking of the $\mathrm{I}_{\mathrm{Kr}}$ channel, which results in bradycardia-dependent ventricular arrhythmias [15]. Moreover, the arrhythmogenic potential of the LQTS3 is aggravated during bradycardia [16]. However some experimental data indicate that beta-blockade effectively prevents ventricular arrhythmias in a validated LQTS3 model [17].

The mechanisms, reentry and activity triggered by afterdepolarizations are the underlying cause of acquired long QT syndrome and are related to adrenergic activation. Specific disorders of the ionic currents similar to those resulting from genetic disorders can occur as a result of the action of different medications. The $\mathrm{I}_{\mathrm{Ks}}$ current can be blocked as a result of such anaesthetic agents, i.e. thiopentone, isoflurane or sevoflurane, resulting in an image similar to congenital LQTS1. The $\mathrm{I}_{\mathrm{Kr}}$ current can be blocked by the administration of haloperidol or erythromycin, giving an image similar to the innate LQTS2 [18]. Hypokalemia, hypomagnesemia, hypocalcemia, bradycardia and drugs (e.g. anti-arrhythmic agents from groups IA and III, certain antidepressants, neuroleptics, some antibiotics, some prokinetic agents), heart conditions (ischemia, heart failure, cardiomyopathy, myocarditis), endocrine disorders (diabetes, hypothyroidism or hyperthyroidism), predispose one to the occurrence of arrhythmias by extending the duration of the action potential and the QT interval. Table 2 shows the main causes of acquired LQTS.

The list of drugs that can induce a prolongation of ventricular repolarization, therefore presenting pro-arrythmo-

Table 2. The main causes of acquired LQTS

\begin{tabular}{l} 
Drug-induced acquired LQTS \\
- Anti-arrhythmic drugs \\
- Certain non-sedating antihistamines \\
- Macrolide antibiotics \\
- Certain psychotropic medications \\
- Certain gastric motility agents \\
\hline Metabolic factors: \\
- Electrolyte disturbances (hypokalemia, hypomagnesemia, \\
hypocalcemia) \\
- Impaired hepatic and/or renal function \\
\hline Structural heart disease \\
Bradyarrhythmias (especially complete heart block) \\
Cerebrovascular diseases (stroke, increased intracranial pressure, \\
subarachnoid haemorrhage) \\
Hypothermia \\
Hypothyroidism
\end{tabular}

genic activity is constantly updated and available on the following website: www.crediblemeds.org. It is extremely rare that the mere fact of the prolongation of repolarization leads to an induction of arrhythmia. The initiation and persistence of arrhythmias has a multifactorial etiology [10].

The treatment of patients with congenital and acquired LQTS differs. The therapeutic approach to congenital LQTS begins with medical termination of the sympathetic input to the myocardium with beta blockers. Rare methods include surgical left cervicothoracic sympathectomy, permanent cardiac pacing or implantation of a cardioverter-defibrillator. Acquired LQTS is commonly associated with pauses or bradycardia, in contrast to some forms of congenital LQTS in which arrhythmias often follow a sudden adrenergic stimulation. The management of acquired LQTS involves acute therapy of arrhythmia, discontinuation of any precipitating drug, as well as the correction of metabolic disorders (e.g. hypokalemia, hypomagnesemia).

\section{THE MODIFICATION OF CARDIAC REPOLARIZATION AFTER THE USE OF LOCAL ANAESTHETICS}

Local anaesthetics reversibly inhibit the formation, the propagation and oscillation of electrical impulses in all excitable tissues by blocking the depolarization process of the cell membrane by combining with sodium channel proteins. The deposition of the drug in the desired site of action results in blocking the nerve fibres, as the effect of a high concentration of local anaesthetic. If the drug, at a certain concentration, enters the systemic circulation, its effect becomes generalized. From the clinical point of view, the key impact of toxicity is on the heart and brain. Toxic activity within the cardiomyocytes reduces electrical excitability, conduction velocity and force of contraction, while the intensity of the effect is parallel to the potency of the local anaesthetic drug. The main factors affecting the occurrence of toxic symptoms, in addition to the differences between individual local anaesthetics, are associated with the concentration of the free fraction of the drug in the plasma. This depends on the chemical properties, the applied dose of the drug, the rate of absorption resulting from local perfusion, the method for distribution to other tissues, the degree of protein binding and the rate of metabolism. Methods for depositing local anaesthetics are very diverse, from the application to the skin surface or mucous membranes, as well as through different blocks, namely: infiltration blocks, plexus blocks and single peripheral nerve blocks, spinal nerve root blocks, nerves and sympathetic ganglia blocks until segmental intravenous anaesthesia. Not without significance is also the choice of the drug delivery technique: a single bolus or continuous application. 
When analyzing the available literature, it is apparent that the impact of some local anaesthetic drugs and techniques of regional anaesthesia on the repolarization process of the cardiomyocytes has not been studied sufficiently.

\section{BUPIVACAINE}

In the case of accidental inadvertent intravenous injection, the toxic effect of bupivacaine on cardiac myocyte function refers to the rapid inhibition of both the sodium current $\left(\mathrm{I}_{\mathrm{Na}}\right)$ and calcium current $\left(\mathrm{I}_{\mathrm{Ca-}}\right)$. In addition, in the induction of arrhythmia, the blocking effect on the transient outward potassium current $\left(I_{\text {to }}\right)$ and the rapid component of the delayed potassium current $\left(\mathrm{I}_{\mathrm{Kr}}\right)$ is considered. With regard to patients with baseline repolarization disorders (e.g. in congenital LQTS syndrome), either a shortening or a prolongation of the QT interval can be expected, depending on which of the above mechanism prevails. In the case of congenital LQTS type 1 syndrome, the blocking of the potassium current of the $\mathrm{I}_{\mathrm{Ks}}$ leads to prolonged repolarization, which is then dependent on the rapid component. Therefore, the blockade of the rapid component occurring after exposure to bupivacaine also significantly prolongs repolarization [19]. On the other hand, if the underlying cause of repolarization disorders is the inhibition of the rapid potassium current component, as observed in LQTS 2, then additional prolongation of the repolarization period as a result of bupivacaine will not occur. Thereafter, the blocking effect on the fast sodium channels $\left(I_{\mathrm{Na}}\right)$ and slow calcium channels $\left(I_{C a-L}\right)$ predominates, which gives one the picture of the shortening of the duration of the activation potential. Moreover, it has been shown that the ability to block potassium currents relates rather to the Ito and $I_{\mathrm{Kr}}$ channels. In contrast, the $I_{\mathrm{Ks}}$ and $\mathrm{I}_{\mathrm{K} 1}$ channels tend to be insensitive to the action of bupivacaine. Therefore, the final effect is the sum of the above-described varying degrees of influence on the ionic currents and depends on the concentration of bupivacaine in the blood. According to some reports, an intravenous administration of bupivacaine does not extend the period of repolarization (no changes in the QT interval, Tp-e) [20]. The difference in pathophysiology between bupivacaine (mainly arrhythmogenic) and lidocaine (which, in therapeutic doses, has minimal negative inotropic effects) should be taken into account.

\section{LIDOCAINE}

Class Ib drugs, according to the Vaughan-Williams classification of anti-arrhythmic drugs, which includes, among others, lidocaine, selectively bind themselves to sodium channels in the inactivated state, causing a reduction of the repolarization duration. In cases of congenital (especially LQTS 3) or acquired prolongation of repolarization, lidocaine is considered to possess a preventive action in relation to the occurrence of ventricular tachycardia (TdP), although its sole effect on repolarization is small [21, 22].

\section{OTHER LOCAL ANAESTHETICS}

There are few studies that have examined the effect of the newer local anaesthetics (levobupivacaine, ropivacaine) on cardiac repolarization [23-25]. Comparative analysis of the effects of bupivacaine and ropivacaine in relation to their impact on the myocardial repolarization in humans has demonstrated that ropivacaine is a safe alternative for the current intraventricular conduction disorder [26, 27]. However, the results of research conducted using levobupivacaine and bupivacaine for spinal anaesthesia in patients undergoing caesarean section were inconclusive. There are reports of non-inferiority and of the advantages of a particular drug, even in cases of long QT syndrome, as well as an important extension of the QT interval after the application of levobupivacaine when compared to bupivacaine. Moreover, in these cases it must be emphasized that QT interval is prolonged during normal pregnancy: regarding certain pathologies (pre-eclampsia), and after the application of oxytocin [28-34].

\section{THE EFFECT OF REGIONAL ANAESTHESIA TECHNIQUES ON THE PERIOD OF REPOLARIZATION}

It is important to determine the range of blocking adrenergic fibres. Stimulation of adrenergic activity through thoracic fibres (T1-4) prolongs repolarization and increases its dispersion [35, 36]. Blocking the sympathetic fibres with epidural anaesthesia and T1-T4 covering the chronotropic effect, leads to a shortening of the QT interval and a reduction in dispersion. What also remains important is the impact of baroreceptor stimulation and the effect of hypotension resulting from extensive sympathetic blockade. The adrenergic blockade as a result of spinal or epidural, not including T1-4 fibres, causes severe adrenergic activity above the level of the block and consequently prolongs repolarization $[37,38]$.

Left-sided stellate ganglion blockade may even lead to a shortening of the QT interval $[39,40]$. Left cardiac sympathetic denervation - LCSD's antifibrillatory effect - has been well described. Indeed, it is both, a safe and effective treatment option to reduce life-threatening ventricular arrhythmias in patients with heritable channelopathies such as LQTS. A prophylactic LCSD, rather than a prophylactic ICD, may represent a good treatment option for patients with unacceptable $\beta$-blocker-related side effects [41]. In contrast, a stellate ganglion blockade on the right side significantly prolongs the QT interval while the QT dispersion increases. 
With respect to the brachial plexus, blocked access between the oblique muscles, regardless of the drug, did not interfere with the duration of repolarization [42].

No results have been found regarding the impact of intravenous anaesthesia techniques on cardiac repolarization in humans. In animal studies, after the intravenous administration of bupivacaine, mepivacaine and etidocaine, QT prolongation, QT dispersion and T wave morphology changes were observed [43].

\section{RECOMMENDATIONS FOR THE MANAGEMENT OF PATIENTS WITH CONGENITAL OR ACQUIRED LQT SYNDROME FOR PLANNED REGIONAL ANAESTHESIA}

There are no guidelines for the perioperative management of a patient with confirmed long QT syndrome [44]. Therefore, spinal or epidural anaesthesia, peripheral nerve block and intravenous regional anaesthesia may be used. However, the concomitant drugs which can prolong repolarization should be avoided. The following opinion may only be used for guidance in these situations. A patient with documented LQT syndrome requires special vigilance by the therapeutic team. The significant prevention of arrhythmias is optimal perioperative management (avoiding acid-base balance disturbances, avoiding ion deficiency — namely potassium, magnesium and calcium — avoiding hypothermia). The therapeutic principles for acquired or congenital LQTS are different. Please note that for $25 \%$ to $40 \%$ of patients with congenital LQT, the 12-lead ECG reading is not diagnostic. Therefore, the anaesthetist should focus on the past medical history of sudden unexplained episodes of loss of consciousness or sudden death in the family.

During perioperative care, adrenergic stimulation resulting from stress and pain should be prevented (appropriate premedication, adequate analgesia). Secure direct access to the resuscitation equipment is obligatory in case of a high risk of TdP. The predisposing factors for TdP include: female sex, bradycardia, electrolyte disturbances (hypokalemia, hypomagnesemia, hypocalcemia), heart failure, left ventricular hypertrophy, subclinical LQTS, some polymorphisms of genes encoding ion channel proteins or proteins associated with the channels relevant to myocardial ventricular repolarization. In the case of arrhythmia induction in the perioperative period, the therapeutic management should be implemented according to the mandatory guidelines of the local heart society.

\section{SUMMARY}

Regardless of the reasons for the prolongation of the repolarization period (congenital, acquired), vigilance is required in perioperative anaesthetic management so as not to lead to the occurrence of ventricular arrhythmias.
Regional anaesthesia techniques and properly applied local anaesthetic drugs are regarded as safe in these patients.

\section{ACKNOWLEDGEMENTS}

1. The authors declare no financial disclosure.

2. The authors declare no conflict of interest.

\section{References:}

1. Zaręba W, Couderc JP: Repolarization morphology and variability mechanisms and clinical application. In: Sobieszczańska M. (ed.): Electrocardiology 2009. JAKS Publishing Company 2010: 57-66.

2. Roden $D$, Abraham $R$ : Refining repolarization reserve. Heart Rhythm 2011; 8: 1756-1757. doi: 10.1016/j.hrthm.2011.06.024.

3. Hekkala A-M, Väänänen $H$, Swan H, Oikarinen L, Viitasalo M, Toivonen L: Reproducibility of computerized measurements of QT interval from multiple leads at rest and during exercise. Ann Noninvasive Electrocardiol 2006; 11: 318-326.

4. Porthan $K$, Viitasalo $M$, Jula A et al.: Predictive value of electrocardiographic QT interval and T-wave morphology parameters for all-cause and cardiovascular mortality in a general population sample. Heart Rhythm 2009; 6: 1202-1208.

5. Rautaharju P, Surawicz B, Gettes L: AHA/ACCF/HRS Recommendations for the Standardization and Interpretation of the Electrocardiogram: Part IV: The ST Segment, T and U Waves, and the QT Interval A Scientific Statement From the American Heart Association Electrocardiography and Arrhythmias Committee, Council on Clinical Cardiology; the American College of Cardiology Foundation; and the Heart Rhythm Society Endorsed by the International Society for Computerized Electrocardiology. JACC 2009; 5: 982-991 doi: 10.1016/ j.jacc.2008. 12.01.

6. Malik M, Batchvarov VN: Measurement, interpretation and clinical potential of QT dispersion. J Am Coll Cardiol 2000; 36: 1749-1766.

7. Oikarinen L, Paavola M, Montonen J et al. Magnetocardiographic QT interval dispersion in postmyocardial infarction patients with sustained ventricular tachycardia: validation of automated OT measurements. Pacing Clin Electrophysiol 1998; 21: 1934-1942.

8. Gupta P, Patel C, Patel Het al.: T(p-e)/QT ratio as an index of arrhythmogenesis. J Electrocardiol 2008; 41: 567-574. doi: 10.1016/j.jelectrocard.2008.07.016.

9. Porthan $K$, Viitasalo $M$, Toivonen L et al: Predictive value of electrocardiographic T-wave morphology parameters and T-wave peak to T-wave end interval for sudden cardiac death in the general population. Circ Arrhythm Electrophysiol 2013; 6: 690-696. doi: 10.1161/ CIRCEP.113.000356.

10. Staikou C, Stamelos M, Stavroulakis E: Impact of anaesthetic drugs and adjuvants on ECG markers of torsadogenicity. Br J Anaesth 2014; 112: 217-230. doi: 10.1093/bja/aet412.

11. Arking DE, Pulit SL, Crotti L et al.: Genetic association study of QT interval highlights role for calcium signaling pathways in myocardial repolarization. Nat Genet 2014; 46: 826-836. doi: 10.1038/ng.3014.

12. Shryock JC1, Song Y, Rajamani S, Antzelevitch C, Belardinelli L: The arrhythmogenic consequences of increasing late $\mathrm{I}_{\mathrm{Na}}$ in the cardiomyocyte. Cardiovasc Res 2013; 99: 600-611. doi: 10.1093/cvr/cvt145.

13. Wu L, Ma J, Li H et al.: Late sodium current contributes to the reverse rate-dependent effect of $\mathrm{IKr}$ inhibition on ventricular repolarization. Circulation 2011; 123: 1713-1720. doi: 10.1161/CIRCULATIONAHA. 110.000661.

14. Argelia Medeiros-Domingoa A, Iturralde-Torresb P, Ackermanc M: Clinical and Genetic Characteristics of Long QT Syndrome. Rev Esp Cardiol 2007; 60: 739-752. doi: 10.1016/S1885-5857(08)60010-9.

15. Osadchii OE: Effects of $\mathrm{Na}+$ channel blockers on extrasystolic stimulation-evoked changes in ventricular conduction and repolarization. J Cardiovasc Pharmacol 2014; 63: 240-251. doi: 10.1097/ FJC.0000000000000041.

16. Bankston JR, Kass RS: Molecular determinants of local anesthetic action of beta-blocking drugs: Implications for therapeutic management of long QT syndrome variant 3. J Mol Cell Cardiol 2010; 48: 246-253. doi: 10.1016/j.yjmcc.2009.05.012.

17. Calvillo L, Spazzolini C, Vullo E, Insolia R, Crotti L, Schwartz PJ: Propranolol prevents life-threatening arrhythmias in LQT3 transgenic mice: implications for the clinical management of LQT3 patients. Heart Rhythm 2014; 11: 126-123. doi: 10.1016/j.hrthm.2013.10.029. 
18. Owczuk R, Wujtewicz MA, Zienciuk-Krajka A, Lasińska-Kowara M, Piankowski A, Wujtewicz M: The influence of anesthesia on cardiac repolarization. Minerva Anestesiol 2012; 78: 483-495.

19. Siebrands C, Binder S, EckhoffU, Schmitt N, Friederich P: Long QT 1 mutation KCNQ1 A344V increases local anesthetic sensitivity of the slowly activating delayed rectifier potassium current. Anesthesiology 2006; 105: 511-520.

20. Schwoerer AP, Zenouzi $R$, Ehmke $H$, Friederich P: Bupivacaine destabilizes action potential duration in cellular and computational models of long QT syndrome 1. Anesth Analg 2011;113:1365-1373. doi: 10.1213/ ANE.0b013e3182323245.

21. Paul AA, Witchel HJ, Hancox JC: Inhibition of the current of heterologously expressed HERG potassium channels by flecainide and comparison with quinidine, propafenone and lignocaine. Br J Pharmacol 2002; 136: 717-729.

22. Owczuk R, Wujtewicz MA, Sawicka W et al.: The effect of intravenous lidocaine on QT changes during tracheal intubation. Anaesthesia 2008; 63: 924-931. doi: 10.1111/j.1365-2044.2008.05525.x.

23. Stewart J, Kellett N, Castro D: The central nervous system and cardiovascular effects of levobupivacaine and ropivacaine in healthy volunteers. Anesth Analg 2003; 97: 412-416.

24. Knudsen K, Beckman Suurküla M, Blomberg S, Sjövall J, Edvardsson $N$. Central nervous and cardiovascular effects of i.v. infusions of ropivacaine, bupivacaine and placebo in volunteers. Br J Anaesth 1997; 78: 507-514.

25. Moller R, Covino BG: Cardiac electrophysiologic properties of bupivacaine and lidocaine compared with those of ropivacaine, a new amide local anaesthetic. Anesthesiology 1990; 72: 322-329.

26. Eledjam JJ, de la Coussaye JE, Colson $P$ et al.: Is epidural anaesthesia using bupivacaine safe in patients with atrio-ventricular conduction defects? Acta Anaesthesiol Scand 1989; 33: 402-404.

27. Güven O, Sazak H, Alagöz A et al.: The effects of local anaesthetics on QT parameters during thoracic epidural anaesthesia combined with general anaesthesia: ropivacaine versus bupivacaine. Balkan Med J 2013; 30: 410-414. doi: 10.5152/balkanmedj.2013.9275.

28. Deniz Y, Okyay D, Hancı V et al.: The effect of levobupivacaine and bupivacaine on QT, corrected QT (Qtc), and P wave dispersions in cesarean section. Braz J Anesthesiol 2013; 63: 202-208. doi: 10.1016/S00347094(13)70216-2.

29. Al-Refai A, Gunka V, Douglas J: Spinal anesthesia for Cesarean section in a parturient with long QT syndrome. Can J Anaesth 200; 51: 993-996.

30. Kameyama $E$, Ito $Y$, Ito $\mathrm{J}$ : Anesthetic management of caesarean section in a patient with asymptomatic idiopathic prolonged QT interval syndrome. Masui 2004; 53: 1167-1169.

31. Dogan Z, Yildiz H, Akcay A: The effect of intraspinal bupivacaine versus levobupivacaine on the QTC intervals during caesarean section: a randomized, double-blind, prospective study. Basic Clin Pharmacol Toxicol 2014; 114: 248-253. doi: 10.1111/bcpt.12146.

32. Guillon A, Leyre S, Remérand F et al.: Modification of Tp-e and QTc intervals during caesarean section under spinal anaesthesia. Anaesthesia 2010; 65: 337-342. doi: 10.1111/j.1365-2044.2010.06246.x.
33. Hodgkinson P, McAtamney D: Combined spinal-epidural anaesthesia for caesarean section in a patient with long QT syndrome. Int J Obstet Anesth 2008; 17: 78-80. doi.org/10.1016/j.ijoa.2007.03.002.

34. Sen S, Ozmert G, Turan H, Caliskan E, Onbasili A, Kaya D: The effects of spinal anesthesia on QT interval in preeclamptic patients. Anesth Analg 2006; 103: 1250-1255.

35. Schwartz PJ, Priori S, Cerrone M et al.: Left cardiac sympathetic denervation in the management of high risk patients affected by the long QT syndrome. Circulation 2004; 109: 1826-1833.

36. Owczuk R, Steffek M, Wujtewicz MA, et al. Effects of thoracic epidural anaesthesia on cardiac repolarization. Clin Exp Pharmacol Physiol 2009; 36: 880-883. doi: 10.1111/j.1440-1681.2009.05163.x.

37. Wang $X$, Chen GY, Yang SS et al.: Effects of high thoracic epidural anesthesia on ischemic cardiomyopathy cardiac function and autonomic neural function. Genet Mol Res 2014; 13: 6813-6819. doi: 10.4238/2014.

38. Owczuk R, Sawicka W, Wujtewicz MA, Kawecka A, Lasek J, Wujtewicz M Influence of spinal anesthesia on corrected QT interval. Reg Anesth Pain Med 2005; 30: 548-552.

39. Fujii K, Yamaguchi S, Egawa H, Hamaguchi S, Kitajima T, Minami J: Effects of head-up tilt after stellate ganglion block on QT interval and QT dispersion. Reg Anesth Pain Med. 2004; 29: 317-322.

40. Milne JR, Ward DE, Spurrell RA, Camm AJ:The long QT syndrome; effects of drugs and left stellate ganglion block. Am Heart J 1982; 104: 194-198.

41. Bos JM, Bos KM, Johnson J, Moir Ch, Ackerman M: Left Cardiac Sympathetic Denervation in Long QT Syndrome. Analysis of Therapeutic Nonresponders. Circ Arrhythm Electrophysiol 2013; 6: 705-711. doi: 10.1161/CIRCEP.113.000102.

42. Borgeat A, Ekatodramis $G$, Blumenthal S: Interscalene brachial plexus anesthesia with ropivacaine $5 \mathrm{mg} / \mathrm{mL}$ and bupivacaine $5 \mathrm{mg} / \mathrm{mL}$ : effects on electrocardiogram. Reg Anesth Pain Med. 2004; 29: 557-563.

43. Kasten GW: Amide local anaesthetic alterations of effective refractory period temporal dispersion: relationship to ventricular arrhythmias. Anesthesiology 1986; 65: 61-66.

44. Staikou C, Chondrogiannis $K$, Mani A. Perioperative management of hereditary arrhythmogenic syndromes. Br J Anaesth 2012; 108: 730-744. doi: 10.1093/bja/aes105.

\section{Corresponding author:}

Jowita Biernawska MD, PhD

Department of Anaesthesia, Intensive Care and Acute Poisoning

Pomeranian Medical University

Al. Powstańców WIkp. 72

70-111 Szczecin, Poland

e-mail: lisienko@wp.pl

Received: 2.05.2015

Accepted: 9.03.2016 